\title{
Rapid diagnosis of bacteremia in adults using acridine orange stained buffy coat smears
}

Mark Miller, MD, JACK MENDELSON, MD

\begin{abstract}
The use of acridine orange stained buffy coat smears was assessed as a rapid screening test for bacteremia in adults. A total of 356 consecutive blood cultures were submitted with simultaneous anticoagulated blood samples, from which a buffy coat smear was prepared and stained with acridine orange $(100 \mathrm{mg} / \mathrm{L}$; pH 3.0). Forty-one of 356 blood samples (12\%) yielded organisms in the blood culture system. Compared to blood culture, the overall sensitivity of acridine orange
\end{abstract}

stained buffy coat smears was $16 \%$, specificity $88 \%$, and positive predictive value $13 \%$. There was no statistically significant difference in performance of the test among patients who had fever greater than $39^{\circ} \mathrm{C}$ and/or shock. The low sensitivity and specificity of the test makes it unsuitable as a means of rapid screening for adults with suspected bacteremia. Can J Infect Dis 1990;1(1):7-10

Key Words: Acridine orange, Leukocytes, Septicemia
TN A PATIENT WITH SUSPECTED BACTEREMIA, RAPID demonstration of circulating organisms in the blood, identification of the pathogen, and prompt treatment with appropriate antimicrobial drugs are essential for optimal care. The time between patient blood sampling and initial detection of infectious agents has been shown to have important prognostic significance (1).

Department of Microbiology. McGill University: and Division of Infectious Diseases (Department of Medicine), Jewish General Hospital, Montreal, Quebec

Correspondence and reprints: Dr Mark Miller, Department of Microbiology. Montreal General Hospital. 1650 Cedar Avenue, Montreal, Quebec H3G 1A4. Telephone (514) 934-8074

This paper was presented in part at the annual meeting of the Association of Medical Microbiologists of Quebec. held in June 1988 in Quebec City. Quebec

Received for publication December 20, 1989. Accepted March 2, 1990
Standard detection of bacteremia requires blood sampling from the patient, inoculation of the sample into any of the currently available blood culture systems, an incubation time of hours to days during which the organisms multiply, and detection of growth by various mechanisms. Innumerable studies have evaluated methods of decreasing the time to detection of positive blood cultures. All of these methods rely on active replication of the organisms present in the culture system with subsequent detection of either organism density, nutrient consumption, or metabolite production (2). Therefore, detection of bacterial growth may take from several hours to several days.

Faster confirmation of bacteremia is desirable because of serious sequelae in patients with circulating microorganisms, particularly in the immunocompromised, the elderly, and patients with 
multiple organ dysfunction. Rapid detection methods devised thus far have depended on the identification of circulating microbial components in serum (3-5), or the direct visualization of microorganisms in buffy coat smears from peripheral blood samples using a variety of nonfluorescent staining techniques (6-8).

In one prospective study, evaluation of acridine orange stained buffy coat smears in 89 neonates was able to identify quickly eight of nine episodes of clinical septicemia (9). The test had an overall sensitivity of $88 \%$, a specificity of $96 \%$, and a positive predictive value of $67 \%$. The authors decided to evaluate this method in an adult population.

\section{MATERIALS AND METHODS}

Over a five week period at the Sir Mortimer B Davis/Jewish General Hospital, the role of acridine orange stained buffy coat smears in diagnosing bacteremia in adults was evaluated. Physicians and nurses responsible for performing blood cultures were made aware of the study through posters and ward meetings. They were instructed that, in any patient with suspected bacteremia on whom blood cultures were being performed. blood was to be routinely inoculated as usual into one aerobic and one anaerobic blood culture bottle (Frappier Diagnostic Inc, and Fisher Scientific, respectively). However, they were asked to place simultaneously an extra 5 to $7 \mathrm{~mL}$ of blood in a sterile tube containing EDTA, available in all areas of the hospital. At the laboratory, the specimen in EDTA was kept at $4^{\circ} \mathrm{C}$ until the buffy coat smear was made (a minimum of 30 mins and a maximum of $10 \mathrm{~h}$ after collection); the blood culture bottles were processed by the standard hospital technique. This consisted of incubation of both aerobic and anaerobic bottles at $35^{\circ} \mathrm{C}$ for one week prior to discarding as negative, with blind Gram stains and subcultures onto both chocolate and 5\% sheep blood agar (in 5\% carbon dioxide and anaerobically, respectively) on days 1 and 5 of incubation; and daily visual inspection of bottles for turbidity. Visibly turbid bottles were immediately Gram-stained and subcultured as above.

The specimen in EDTA was processed to obtain a buffy coat smear by a modification of the method of Brooks et al (7). In brief, the blood was centrifuged at $700 \mathrm{~g}$ for $10 \mathrm{mins}$ and the serum removed with a sterile Pasteur pipette. The buffy coat was then removed with a sterile pipette and two drops were spread on a sterile glass slide. The slides were marked with numbers only, in order to blind the microscopist as to the origin of the smear.

The smears were air dried, heat fixed, and subsequently stained with acridine orange at a con- centration of $100 \mathrm{mg} / \mathrm{L}$ buffered at $\mathrm{pH} 3.0$ according to the method of Kronvall and Myhre (10). The solution was overlaid on the slides for a duration of 2 mins. The slides were then rinsed with distilled water for $15 \mathrm{~s}$, and air dried.

The smears were read within $1 \mathrm{~h}$ of staining on a fluorescent microscope using 630x magnification. Suspicious fluorescence was confirmed using $1000 x$ magnification. The buffy coat smears were graded 'positive' if one or more bacteria or fungi were visualized per slide. Each smear was also graded on the basis of the number of white blood cells present: 'leukocyte-poor' if less than 10 white blood cells were seen per $630 x$ field, and 'leukocyte-rich' otherwise. In order to standardize the microscopy, each slide was read for 5 mins. One investigator (MM) read all the smears.

Sensitivity, specificity and positive and negative predictive values of the test were calculated using the standard formulae (11). Confidence intervals for proportions were calculated assuming a binomial distribution (12).

\section{RESULTS}

Three hundred and fifty-six consecutive blood samples were received in the laboratory for inclusion in the study. The distribution of patient locations was as follows: medical or surgical wards 195 (55\%), emergency room 148 (41\%), and intensive care units $13(4 \%)$

Forty-one of the 356 blood samples (12\%) yielded positive blood cultures. The distribution of organisms was as follows: aerobic Gram-negative rods $28(68 \%)$, aerobic Gram-positive cocci 10 $(25 \%)$, anaerobes two $(5 \%)$, yeast one $(2 \%)$, and mixed zero. Three of the 10 samples which yielded Gram-positive cocci grew Staphylococcus epidermidis. The three patients were not treated, and the organism was considered by the treating physicians to be a contaminant. Their blood cultures were considered negative for the purpose of this study.

Forty-three specimens yielded 'leukocyte-poor' buffy coat smears, as previously defined. Of these, most originated from patients with absolute leukocyte counts less than $1000 / \mathrm{mm}^{3}(44 \%)$, metastatic cancer or hematologic malignancy $(33 \%)$, or the acquired immune deficiency syndrome $(5 \%)$.

Forty-five buffy coat smears were read as 'positive'. Table 1 shows the distribution of positive blood cultures and buffy coat smears. The operating characteristics of the fluorescent smear are as follows: sensitivity $16 \%(95 \%$ confidence interval 6 to $31 \%$ ), specificity $88 \%$ ( 84 to $92 \%$ ), positive predictive value $13 \%$ (5 to $27 \%$ ), and negative predictive value $90 \%$ ( 86 to $93 \%$ ). 
TABLE 1

\section{Results of blood cultures and buffy coat smears}

\begin{tabular}{lc}
\hline Parameter & Number \\
\hline Positive blood cultures & 38 \\
Positive buffy coat & 6 \\
Negative buffy coat & 32 \\
Negative blood cultures & 318 \\
Positive buffy coat & 39 \\
Negative buffy coat & 279
\end{tabular}

The performance of the test was secondarily analyzed in a group of high risk patients with fever greater than or equal to $39^{\circ} \mathrm{C}$ and/or a diagnosis of shock (systolic blood pressure less than 100 $\mathrm{mmHg}$ and presence of lactic acidosis). The sensitivity of the test in this population was 29\% (95\% confidence interval 8 to $58 \%$ ) which is not significantly different from the overall sensitivity of $16 \%(\mathrm{P}=0.34$; exact binomial test).

There was greater predictive value in finding fluorescent rods than cocci on the smear. Visualization of fluorescent rods correctly predicted Gram-negative rod bacteremia in four of six patients $(67 \%)$, whereas fluorescent cocci were correctly predictive of bacteremia in only two of 39 individuals $(5 \%)$.

\section{DISCUSSION}

It is estimated that a large concentration of organisms $\left(10^{5}\right.$ to $10^{6} / \mathrm{mL}$ of whole blood) must be present in order to be detected with light microscopy (13). Because of this, Gram staining of buffy coats in adults has demonstrated a sensitivity of only $12 \%$ (14). Fluorescent evaluation of such smears has been estimated to be approximately 10 times more sensitive, based on studies comparing fluorescent and Gram stained smears of blood culture bottles (15) or clinical specimens such as cerebrospinal fluid $(16,17)$.

Acridine orange (3,6-bis[dimethylamino] acridine), a basic fluorescent dye, has been shown to bind to both RNA and DNA (18). It does this by intercalating into double stranded chains as well

\section{REFERENCES}

1. Dupont HL, Spink WW. Infections due to Gramnegative organisms: An analysis of 860 patients with bacteremia at the University of Minnesota Medical Center, 1958-1966. Medicine (Baltimore) 1969;48:307-32.

2. Reller LB, Murray PR, MacLowry JD. Cumitech 1A, Blood Cultures II. In: Washington JA, ed. Washington: American Society for Microbiology. 1982:1-11.

3. Levin J, Poore TE, Zauber NP, Oser RS. Detection of endotoxin in the blood of patients with sepsis due to Gram-negative bacteria. N Engl J Med 1970;283:1313-6.

4. de Repentigny L, Reiss E. Current trends in im- as binding to the outside of the double helix (19). In addition to binding to bacterial nucleic acids, it binds to the nucleic acids of fungi, Mycoplasma species (20), trichomonads (21), spirochetes (22), mycobacteria, and malarial parasites (23). There is a striking difference between the fluorescence of the organisms noted above (bright orange) and that of somatic cells (green or yellow) when the stain is buffered at pH 3 to 4 (10). This differential staining was the basis for assessment of its usefulness in detecting pathogens in buffy coat smears.

The results of this study show that an acridine orange stained buffy coat smear has limited clinical usefulness as a screening test in an adult hospital setting because of its low sensitivity. The overall sensitivity of $16 \%$ is similar to that of the Gram-staining of buffy coats previously reported (14), and is only slightly higher than the $11 \%$ prior probability of bacteremia in the authors' adult patients. The test's sensitivity probably results from the low number of bacteria that normally circulate in most bacteremic adults (24).

The low specificity of the test can be attributed to the 39 'positive' buffy coat smears from nonbacteremic patients which displayed fluorescence that resembled bacteria. The probable reason for the test's low specificity is fluorescence of round intracytoplasmic granules which resemble cocci in the leukocytes. These granules fluoresce brightly, probably due to high RNA content. Although on most preparations it was easy to differentiate between coccal bacteria and these granules (the latter being less round in shape, less fluorescent, and a darker orange), many granules sufficiently resembled bacteria to be so mistaken. This accounts for the almost complete predominance of fluorescent 'cocci' seen on false-positive buffy coat smears.

The low sensitivity and specificity of acridine orange stained buffy coat smears in adults with suspected bacteremia makes it unsuitable as a rapid screening test.

munodiagnosis of candidiasis and aspergillosis. Rev Infect Dis 1984:6:301-12.

5. Brooks. JB. Use of frequency-pulsed, electron-capture gas-liquid chromatography to selectively detect certain types of bacterial products produced both in vivo and in vitro. In: Tilton RC, ed. Rapid Methods and Automation in Microbiology. Washington: American Society for Microbiology, 1981:155-61.

6. Faden HS. Early diagnosis of neonatal bacteremia by buffy-coat examination. J Pediatr 1976:88:1032-4.

7. Brooks GF, Pribble AH, Beaty HN. Early diagnosis of bacteremia by buffy-coat examinations. Arch Intern Med 1973:132:673-5.

8. Coppen MJ, Noble CJ, Aubrey C. Evaluation of 
buffy-coat microscopy for the early diagnosis of bacteraemia. J Clin Pathol 1981:34:1375-7.

9. Kleiman MB, Reynolds JK, Schreiner RL, Smith JW, Allen SD. Rapid diagnosis of neonatal bacteremia with acridine orange-stained buffy coat smears. J Pediatr 1984;105:419-21.

10. Kronvall G, Myhre E. Differential staining of bacteria in clinical specimens using acridine orange buffered at low $\mathrm{pH}$. Acta Pathol Microbiol Scand [B] 1977:85:249-54.

11. Ingelfinger JA, Mosteller F, Thibodeau LA, Ware $\mathrm{JH}$. Biostatistics in Clinical Medicine. New York: MacMillan Publishing Co Inc, 1983:4-16.

12. Ingelfinger JA, Mosteller F, Thibodeau LA. Ware JH. Biostatistics in Clinical Medicine. New York: MacMillan Publishing Co Inc, 1983:138-47.

13. McCabe WT, Laporte JJ. Intracellular bacteria in the peripheral blood in staphylococcal bacteraemia. Ann Intern Med 1962;57:141-3.

14. Reik H, Rubin SJ. Evaluation of the buffy-coat smear for rapid detection of bacteremia. JAMA 1981:245:357-9.

15. Mascart G, Bertrand F, Mascart P. Comparative study of subculture, Gram staining and acridine orange staining for early detection of positive blood cultures. J Clin Pathol 1983;36:595-7.

16. Lauer BA, Reller LB, Mirrett S. Comparison of acridine orange and Gram stains for detection of microorganisms in cerebrospinal fluid and other clinical specimens. J Clin Microbiol 1981;14:201-5.

17. Kleiman MB, Reynolds JK, Watts NH, Schreiner RL, Smith JW. Superiority of acridine orange stain versus Gram stain in partially treated bacterial meningitis. J Pediatr 1984;104:401-4.

18. Udenfriend S. Fluorescence Assay in Biology Medicine, Vol 2. New York: Academic Press Inc, 1969:386-8.

19. Le Pecq J-B. Cationic fluorescent probes of polynucleotides. In: Chen RF, Edelhoch H, eds. Biochemical Fluorescence: Concepts, Vol 2. New York: Marcel Dekker Inc, 1976:724.

20. Rosendal S, Valdivieso-Garcia A. Enumeration of mycoplasmas after acridine orange staining. Appl Environ Microbiol 1981;41:1000-2.

21. Harrington BJ, Gaydos JM. Low pH acridine orange stain for trichomonads. Lab Med 1984; 15:180-2.

22. Sciotto CG, Lauer BA, White WL, Istre GR. Detection of Borrelia in acridine orange-stained blood smears by fluorescence microscopy. Arch Pathol Lab Med 1983:107:384-6.

23. Shute GT, Sodeman TM. Identification of malaria parasites by fluorescence microscopy and acridine orange staining. Bull WHO 1973;48:591-6.

24. Tilton RC. The laboratory approach to the detection of bacteremia. Annu Rev Microbiol 1982:36:467-93. 


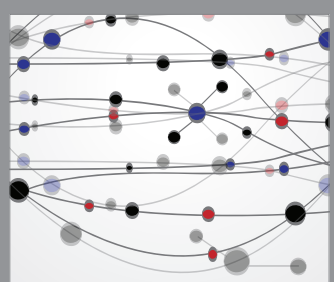

The Scientific World Journal
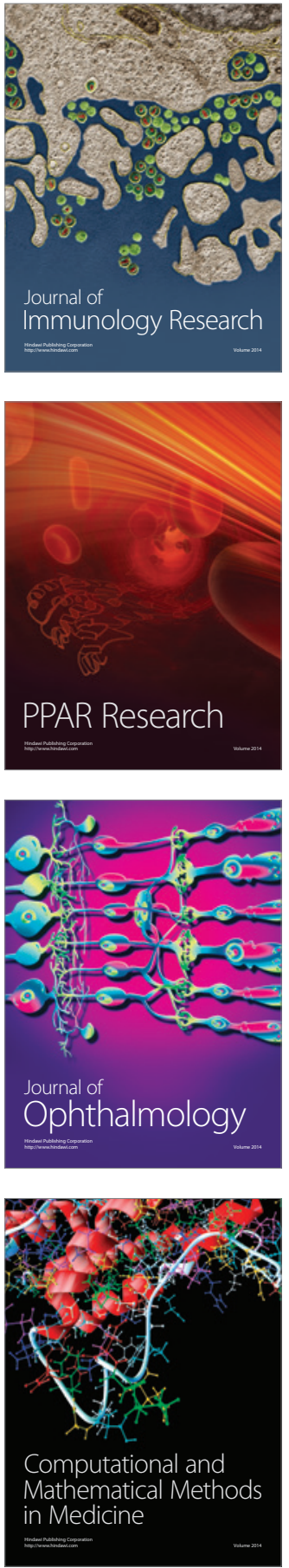

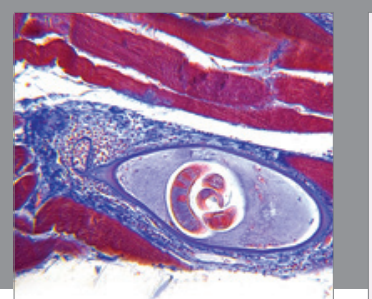

Gastroenterology Research and Practice

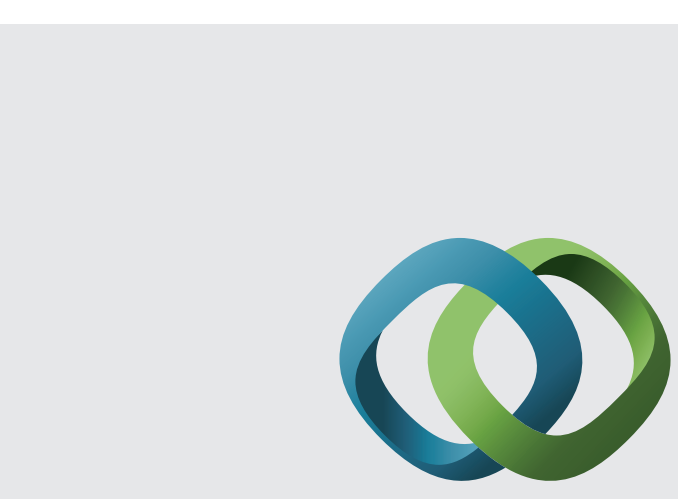

\section{Hindawi}

Submit your manuscripts at

http://www.hindawi.com
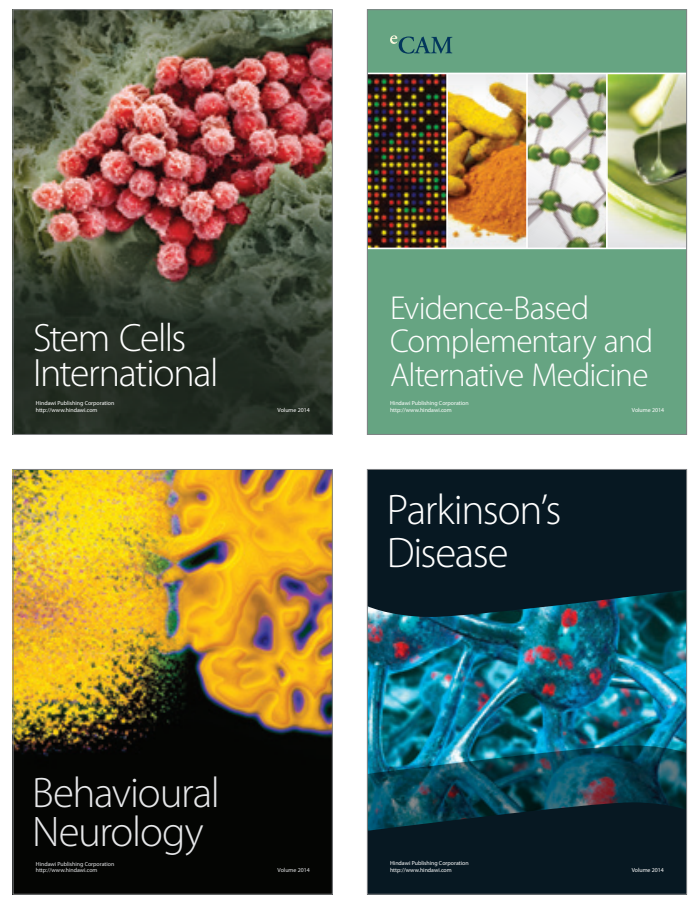
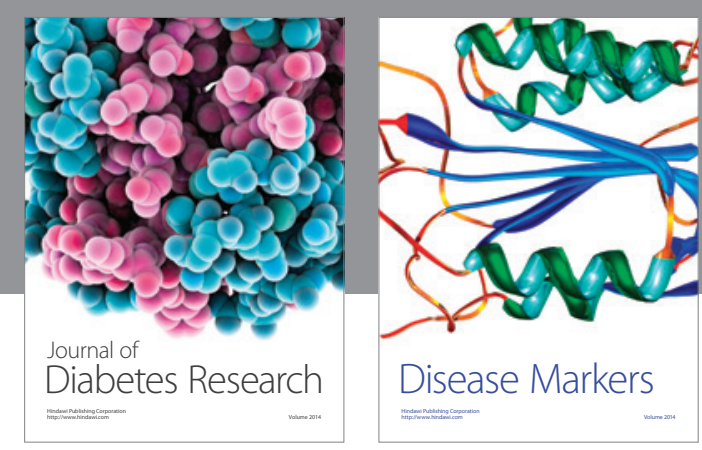

Disease Markers
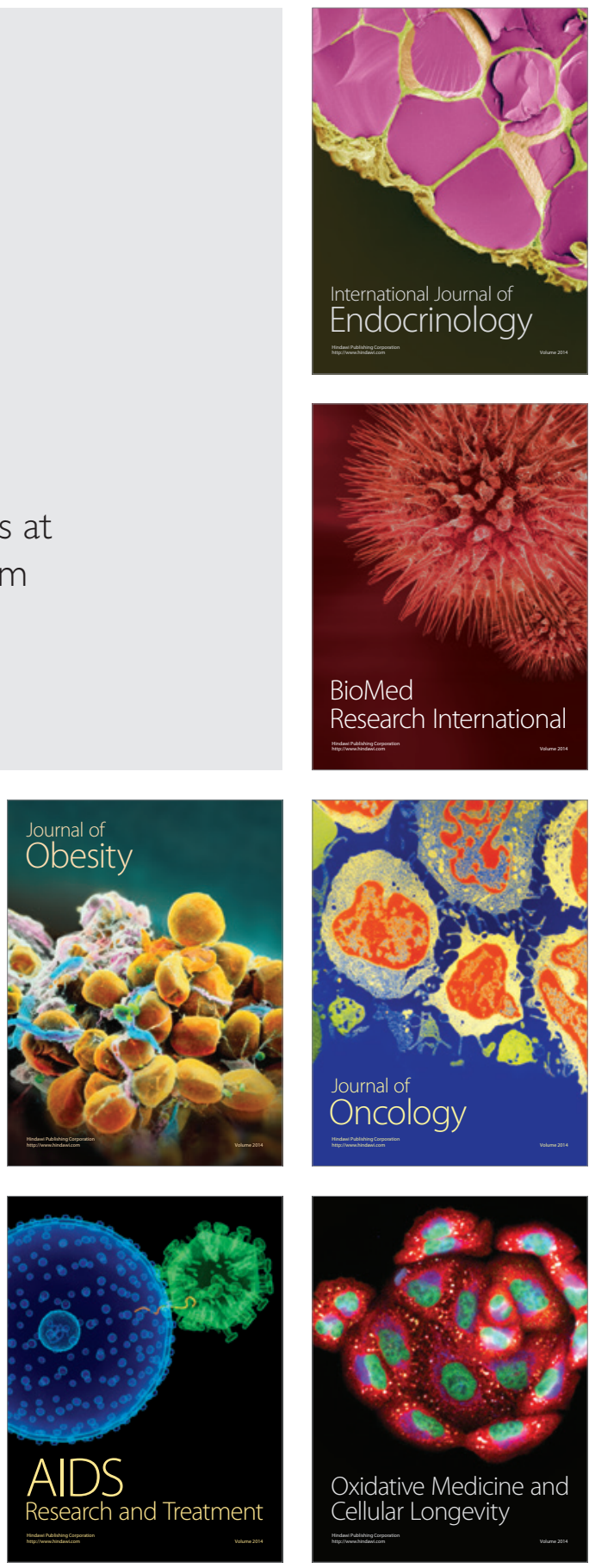